\title{
Impact of in-hospital recurrent ischemia event: findings from GULF RACE-2
}

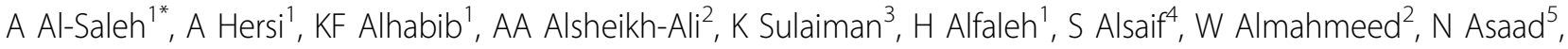 \\ H Amin ${ }^{6}$, A Al-Motarreb $^{7}$, J Al Suwaidi ${ }^{5}$
}

From International Conference for Healthcare and Medical Students 2011

Dublin, Ireland. 4-5 November 2011

\section{Introduction}

Little in the literature is known about the long term outcome of patients with acute coronary syndrome (ACS) and in-hospital recurrent ischemic event. Accordingly; our objectives were to determine the baseline characteristics of patients, the predictors, and the long term outcome of patients with recurrent ischemia.

\section{Methods}

The population compromised 7930 enrolled in the second Gulf Registry of Acute Coronary Events (Gulf RACE-2).

\section{Results}

Out of the 7930 ACS patients, 172 (2.2\%) had recurrent myocardial infarction (Re-MI) during their hospital stay. Patients with Re-MI were more likely to be older (mean age $59.12 \pm 13.5$ vs. $56.8 \pm 12.4, \mathrm{P}=0.016$ ), had significantly higher rate of prior history of angina ( $48 \%$ vs. $38.2 \%$, $\mathrm{P}=0.006)$, and hyperlipidemia ( $45.2 \%$ vs. $37.3 \%, \mathrm{P}=0.027$ ) than patients without Re-MI. On admission patients with Re-MI had significantly higher HR, lower systolic BP, Killip class 4 and high GRACE risk score than those without Re-MI ( $27.3 \%$ vs. $17.6 \%)$, ( $11 \%$ vs. $4.8 \%),(8.1 \%$ vs. $3.2 \%)$, and ( $31.8 \%$ vs. $21.5 \%, \mathrm{P}<0.05$ for all comparisons) respectively. Patients with Re-MI had a higher rate of STEMI on admission than patients without Re-MI (72.1\% vs. $43.9 \%$; $\mathrm{P}<0.001)$. Re-MI patients were less likely to receive Aspirin $(94.8 \%$ vs. $98.5 \%, \mathrm{P}=0.002)$, beta- blockers ( $95.3 \%$ vs. $74.7 \%, \mathrm{P}<0.001)$, and Statin $(87.2 \%$ vs. $94.9 \%$, $\mathrm{P}<0.001)$ than patients without Re-MI. Coronary angiogram was less frequently performed on patients with Re-MI than patients without Re-MI (30.8\% vs. $32.5 \%$,
$\mathrm{P}=0.036)$. In hospital adverse events including HF, cardiogenic shock, VT/VF were more frequent in the Re-MI group than patients without Re-MI (44.2\% vs. $12.4 \%)$, ( $25.6 \%$ vs. $5.3 \%)$, $(7.6 \%$ vs. $2.7 \%$; $\mathrm{P}<0.001$ for all comparisons) respectively. In ACS patients with Re-MI in-hospital, 30 days and 1 year were significantly higher that patients without Re-MI ( $23.8 \%$ vs. $4.1 \%)$, (28.1\% vs. $7.7 \%)$, and (31.6\% vs. $12.1 \% ; \mathrm{P}<0.001$ for all comparisons), respectively.

\section{Conclusions}

Recognizing patients at high risk of Re- MI is important as modifying the risk factors, and managing the patient aggressively may reduce the incidence of such events and the associated morbidity and mortality.

\section{Author details}

'Department of Cardiac Sciences, College of Medicine, King Saud University, Riyadh, Saudi Arabia. ${ }^{2}$ Department of Cardiac Sciences, Sheikh Khalifa Medical City, Abu Dhabi, United Arab Emirates. ${ }^{3}$ Cardiology Department, Royal Hospital, Muscat, Oman. ${ }^{4}$ Cardiology Department, Saud Al-Babtain Cardiac Center, Dammam, Saudi Arabia. ${ }^{5}$ Department of Cardiology and Cardiovascular Surgery, Hamad Medical Corporation (HMC), Doha, Qatar. ${ }^{6}$ Cardiology Department, Mohammed Bin Khalifa Cardiac Center, Manama, Bahrain. 'Department of Medicine, Faculty of Medicine, Sana'a University, Sana'a, Yemen.

Published: 9 July 2012

\section{doi:10.1186/1753-6561-6-S4-017}

Cite this article as: Al-Saleh et al: Impact of in-hospital recurrent ischemia event: findings from GULF RACE-2. BMC Proceedings 20126 (Suppl 4):017. 